$\xi_{\mathrm{N}}$

\title{
Design and implementation of wearable single chip system for sensing, processing and transmitting human gesture and motion data
}

\author{
Venkata Snehith.H*, Likita Ratna D, Syed.Shameem \\ Department of Electronics and Communication Engineering, \\ Koneru Lakshmaiah Education Foundation, Vaddeswaram, India 522502 \\ *Corresponding author E-mail: urssnehith@gmail.com
}

\begin{abstract}
Our work aims at the design of wearable electronic modules that can be assembled as systems for sensing, processing, transmitting, actuating and mimicking human hand gestures and movements, to be used in various robotic, educational, military, medical, industrial, general and hobby applications, the secondary focus of our work is reducing the cost, complexity and assembly of such systems, which are already being used by research centers, laboratories and high-class industries all over the world while the primary objective is to bring these systems down to customizable modular components which could be assembled and combined the way the user wishes to and needs them to be, thereby bringing these concepts closer to a wider range of students, enthusiasts and hobbyists making it easy for them to understand and comprehend these concepts even at the beginner level. In our project, we used commonly available piezo-resistive materials and other household items to make force, pressure, stress, strain and bend sensors and appended them to an Inertial measurement unit, a microcontroller and a wireless transceiver all embedded onto a single chip, to create a simple sensing mechanism that could be worn on a human hand to sense, process and transmit the gestures for actuating and mimicking applications.
\end{abstract}

Keywords: Flexion-Sensing, Inertial Measurement Unit, Motion-Sensing, Processing, Transmitting.

\section{Introduction}

Micro electro mechanical systems or MEMS are a blend of mechanical and electronic systems that allow us to transducer mechanical inputs into electronic outputs. The demand for such systems has rocketed ever since the fields like VLSI, Mechatronics, robotics and industrial machining became popular enough to be adopted into industries and universities, and thereby, to the students and consumer community. These are tiny systems embedded on circuits in the micro scale, that has stationary or movable mechanical systems that respond to the physical and inertial changes, coupled with electronic components that produce an electrical output in correspondence to these changes. MEMS are generally built from mechanical and electronic components that range only up to a $100 \mu \mathrm{m}$ in size, and the systems themselves are typically in size ranges between $20-1000 \mu \mathrm{m}$.

An IMU (inertial measurement unit) is a device that gives the measure of inertia experienced by itself due to any change in its state, caused by an external stimulus. MEMS based IMUs have been trending all over the world, being crucial parts of various devices, gadgets and systems in almost every field of study and industry. Common examples of MEMS based IMUs include Accelerometers, Gyroscopes, Magnetometers, etc. These systems generally include a freely suspending mass (proof), constrained to a motion (or) oscillation in between stationary mechanical system. (spring).
Microcontrollers are essentially the commanding and controlling devices that process the data in electronic and mechatronic systems that demand relatively small to moderate computing capabilities compared to system on chips (SoC). They are designed to carry out calculations, data and memory operations, and handle interrupts, and many more.

Arduino is the best environment available to program the at mega $328 \mathrm{p}$ and develop interesting projects through its open source IDE and libraries. The added advantage of being open source besides free availability is, the vast community that keeps contributing all the time, saving time and effort to beginners and consumers. The highly sophisticated software and hardware offers easy and reliable solution to many programming and electronic challenges. There are many iterations, or revisions and models of the Arduino MUC in the market, depending upon the size and performance requirements.

Transcribing data without the use of any wired media is called wireless communication. Electromagnetic waves are used as carriers of the signal in such cases. Bluetooth, NFC (Near field communication), Wi-Fi (wireless fidelity) and RF (radio frequency) are the commonly used wireless communication standards that serve the purpose efficiently for applications that rely on short distance communications.

One of the two mostly popular standards of wireless communications, used in almost all mobile devices, integrated by default, Bluetooth is quite useful for transcribing data over distances below 100ft. It offers a transfer bandwidth of $800 \mathrm{Kbps}$, and Bit rate 
of $2.1 \mathrm{Mbps}$ with a latency of only $200 \mathrm{~ms}$, allowing it to be ideal for applications where transfer speed and range are not the issue. They operate at Ultra High Frequency band, from 2.408 to $2.485 \mathrm{MHz}$, with four working versions 3.0, 4.0, 5.0 and BLE (Bluetooth low energy). They are simple to connect and can connect to up to seven devices simultaneously.

\section{Principle and Study}

The physical property of certain materials that is responsible for the change in electrical resistance of an object when it is subjected to a mechanical stress, proportional to the strain developed in the object. This is similar to the piezoelectric effect and is only applicable to certain materials called piezo resistive materials like germanium, silicon carbide and almost all forms of crystalline, polycrystalline and amorphous silicon.

\section{A. Study}

In case of semiconductor materials, variation in the spacing between their atoms, due to an external mechanical stress or pressure, bring variations in the band gaps between the conduction and insulation band, thereby, making electronic transitions between the valence and conduction bands easier, leading to changes in the conductivity and therefore the resistivity. The relation between the strain experienced by the material and it's resistivity is linear to a particular extent, which helps us derive the coefficient of piezo-resistivity $\rho_{\sigma}$

$$
\rho_{\sigma}=(\partial \rho / \rho) / \varepsilon
$$

In case of metals, the variation in resistance occurs predominantly because of the geometrical changes in or on the surface of the metal, due to any external force of pressure applied on it, causing a strain to develop from within, which leads to change in the length or area or thickness of the metallic part. In materials whose surface resistance is high enough to contribute a remarkable variation in overall resistance for even slight changes in the geometry, stress, strain, pressure, force can be calculated by:

Strain $\varepsilon=\Delta V / R$, Stress $\mathrm{f}=\Delta \mathrm{V} / \mathrm{RE}$, Pressure $=\mathrm{A} \times \Delta \mathrm{V} / \mathrm{RE}$, Force $\mathrm{F}=\mathrm{A}^{2} \times \Delta \mathrm{V} / \mathrm{RE}$

Flexion/elongation $\Delta L=\Delta \mathrm{V} \times \mathrm{L} / \mathrm{R}$

Hence, the stress, strain, force, pressure, flexion/elongation experienced by the sensor could be measured from the piezo resistivity principle as shown above.

\section{B. Selection of components}

Choosing the right set of components is very crucial in maintaining the compactness and cost effectiveness of the system, without compromising its performance. For the system to be easily understood and adapted by the user community, the components should be very commonly used and known. Hence, the popular Arduino microcontroller, GY-250 IMU, and HC-05 Bluetooth transceiver module are selected to be used with the designed flexsion sensors and the circuit.

\section{Sensor and Circuit Design}

\section{A. Design of flex sensor}

Piezo-resistive flex sensors could be built from a single piezoresistive sheet. Passing a constant current through the sheet from one side and simultaneously measuring the voltage across it's terminals on the other side could give us the variation in the voltage difference at output terminals as a function of variation in resistance due to any mechanical force or pressure applied, which could be later constrained to a range of flexion values with minimum and maximum cut offs, and could be dynamically mapped into angular data.

The construction of the sensor is similar to a simple sandwich, where the piezo-resistive sheet is sandwiched between to conductive metal layers or electrodes that serve as a junction for input power and output voltage difference, as shown below:

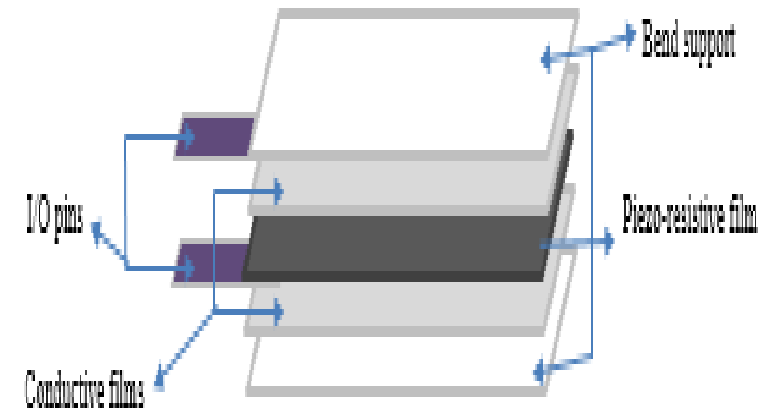

Fig. 1: construction of a cheap velostat flex sensor

\section{B. Circuit design}

The circuit made from all the above-mentioned components is desired to be customizable where the components and connectors are completely replaceable and interchangeable according to the user's wish and necessity. To make this happen, General purpose input, output pins are provided along one side of the circuit, and are connected to the GPIO pins of the Arduino along the analog and digital sides, also, since the Arduino is reusable and reprogrammable, the user can also reprogram the board with a new or updated code, to serve his purpose with the new components. The major area of advancement is the Bluetooth module, since the module operates in a range of not more than $100 \mathrm{ft}$, there is a clear necessity in replacing the Bluetooth with an alternative RF or Wi-Fi transceiver. That is where these general-purpose input and output pins come into play, because the Arduino has a common serial Tx and $\mathrm{Rx}$ port, and a dedicated Serial port for alternative communication, so that two modes of communications could be embedded and later used as per the user's choice. Also, since the circuit is proposed for a wearable application, the design should be very compact despite all it's robustness and power, so, the circuit must be an embedded micro-fabricated and micro-soldered PCB.

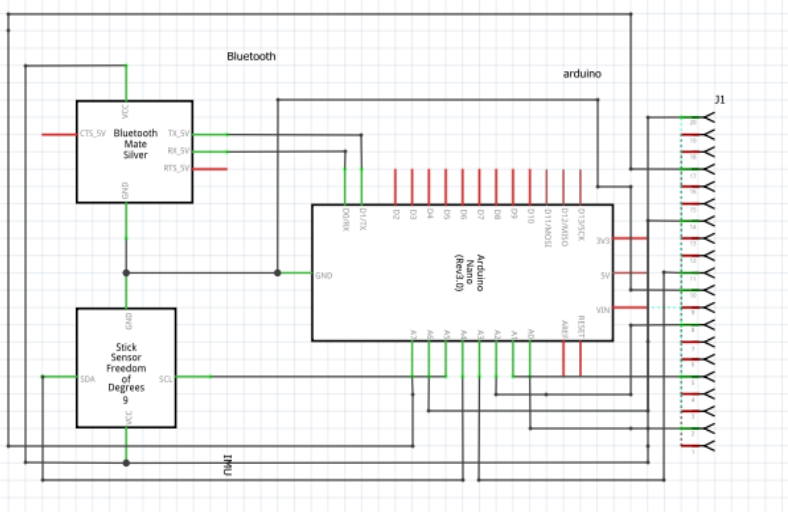

Fig. 2: (a) Circuit schematic design 


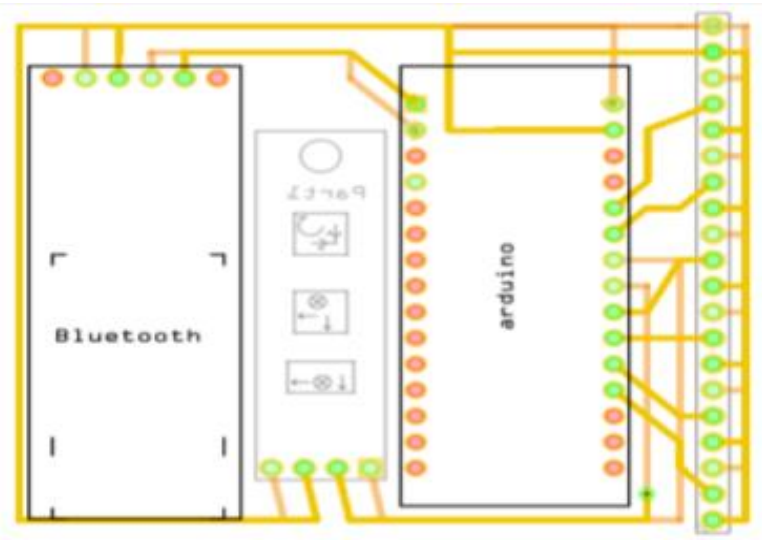

Fig. 2: (b) Initial PCB design

As mentioned in the figures above, the first design iteration (figure 2 (b)) is a less compact version, with dimensions of $2.3622 \times 2.106$ inches (nearly $6 \mathrm{~cm} \times 5.35 \mathrm{~cm}$ ) and could cause minor comfortability issues in wearing, for persons with small wrists. Thus, a new version of the base board is proposed, onto which the components could be micro-soldered is proposed with improved compactness with dimensions of 1.18-inch $\times 1.9685$ inch $(3 \mathrm{~cm} \times 5 \mathrm{~cm})$ which is now suitable for almost all sizes of an adult human hand.

\section{Circuit implementation}

The circuit is coupled with the flex sensors and IMUs and is fabricated in and ergonomic design to be comfortably worn on a human hand, using Velcro straps that provide the required insulation and also comfort.

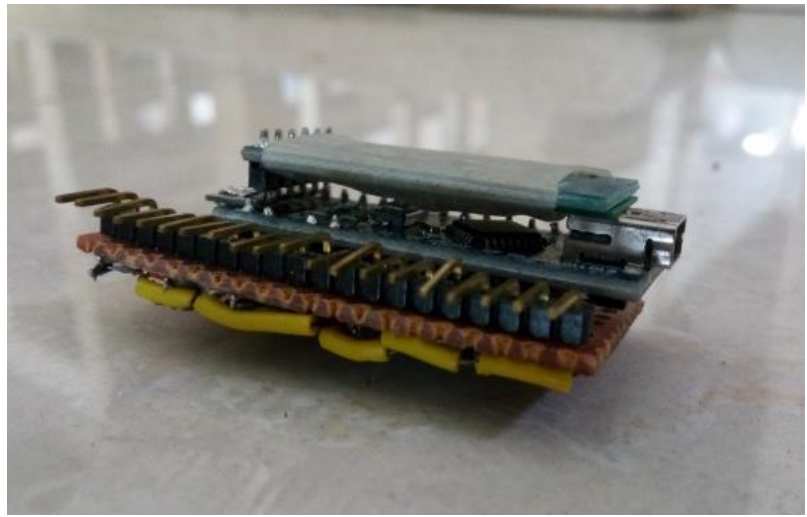

Fig. 3: (a) Hardwired circuit

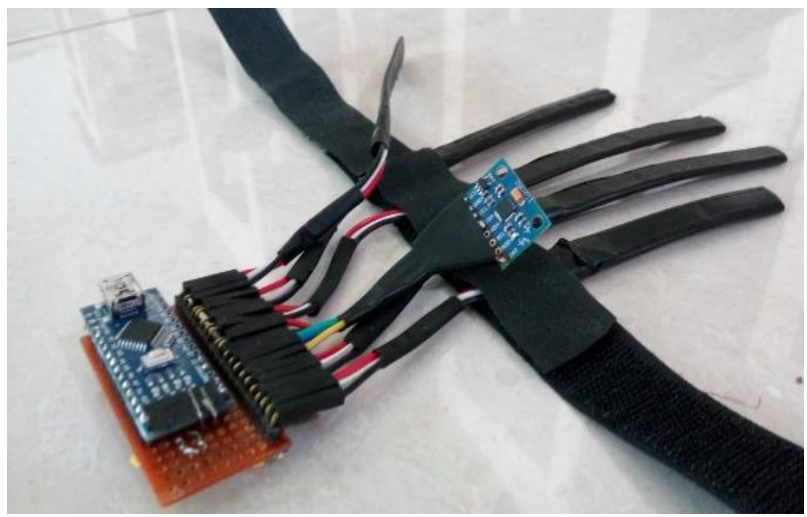

Fig. 3: (b) fully fabricated wearable system

\section{Results}

\section{Flex Sensor Results}

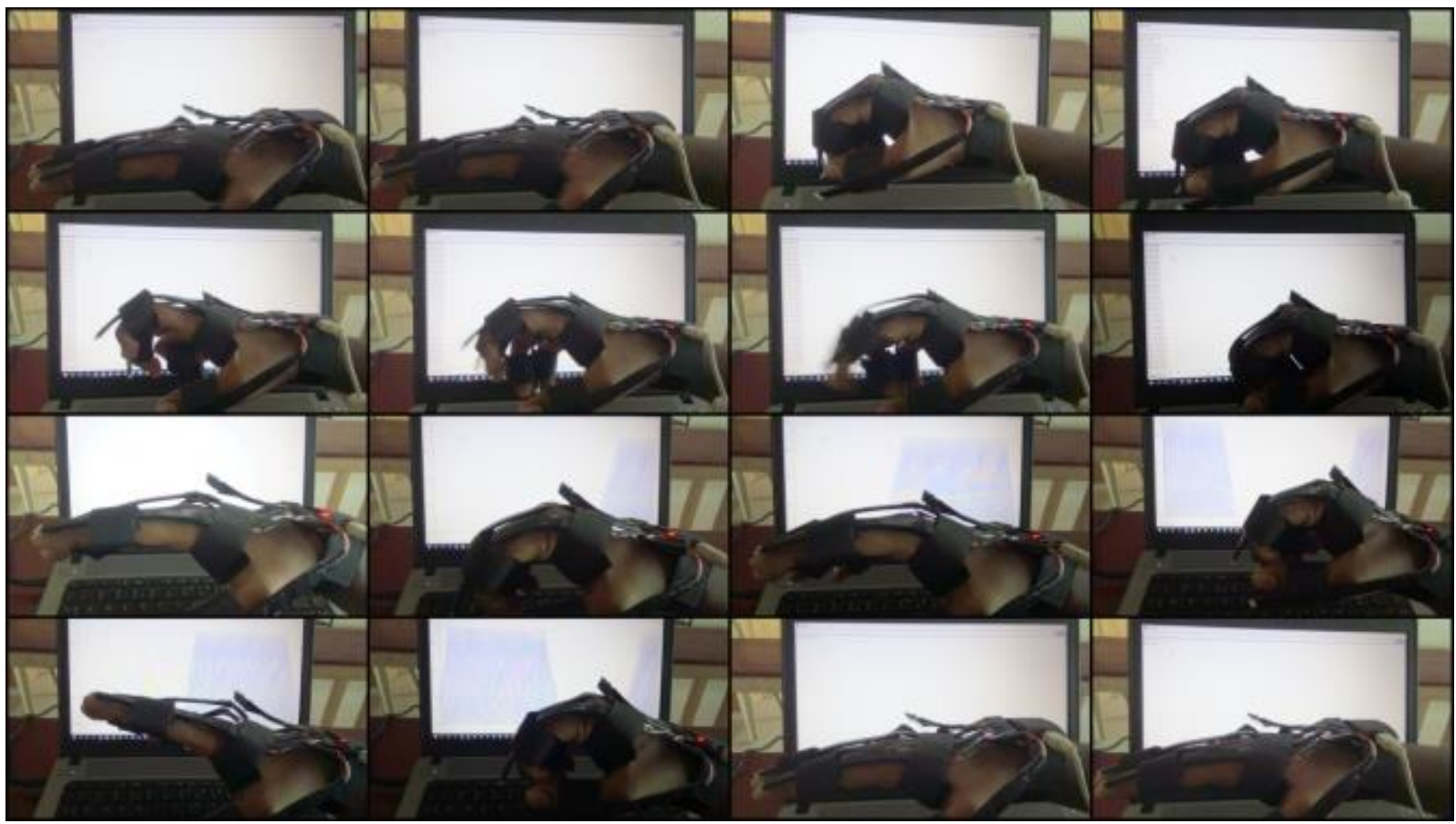

Fig. 4: (a) series of human gestures for simulation testing 


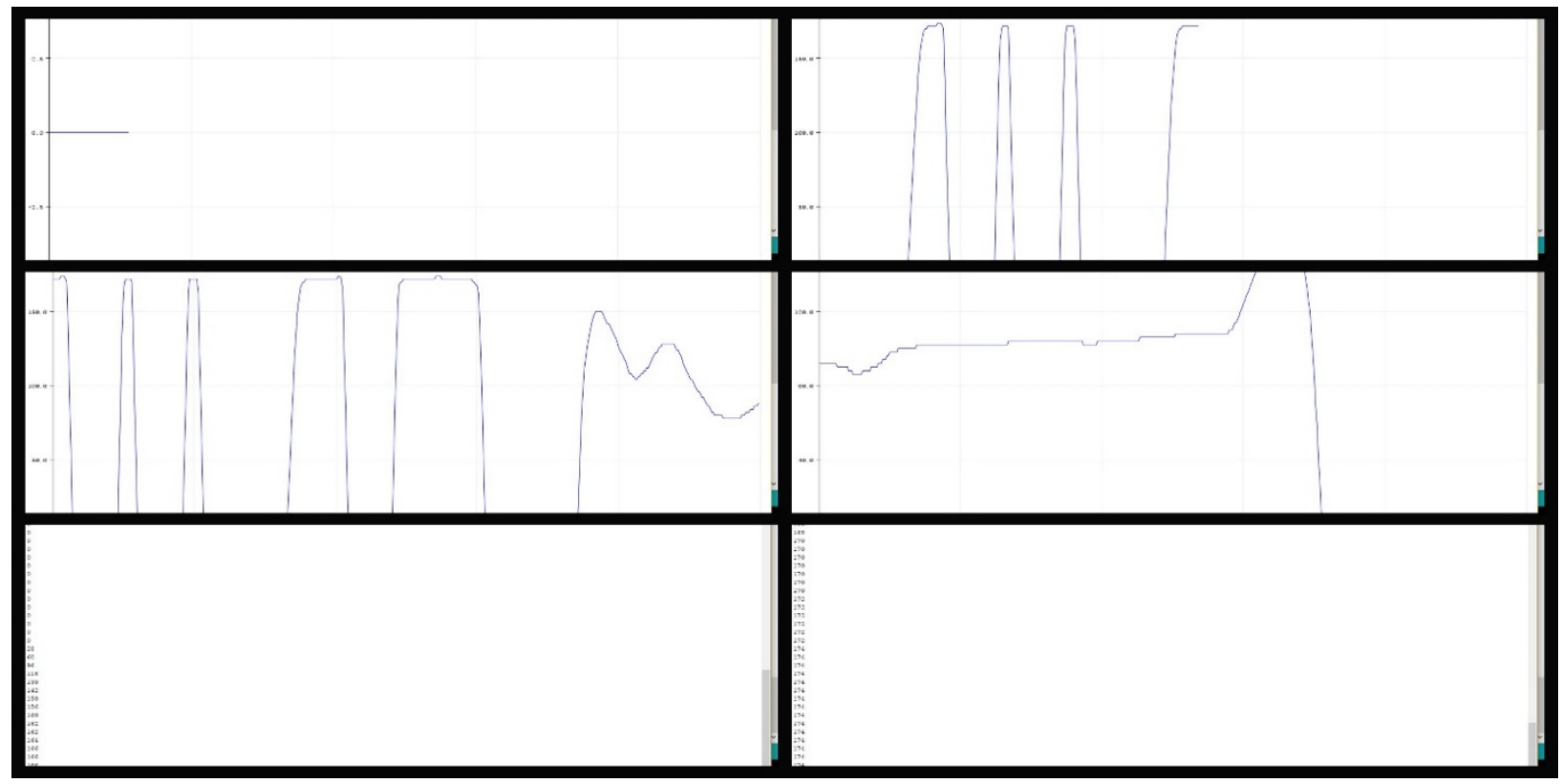

Fig. 4: (b) observed voltage ranges at each sensor node during gestures.

\section{Discussion of results}

The series of gestures of the human hand as shown in figure 4(a) are sensed and graphically represented in figure 4(b) as a plot of the output voltage ranges from flex sensors and the IMU, after a proper calibration, exhibited an accuracy of $96 \%$, with only minor deviations from the actual physical inputs. The detailed comparison of the performance accuracy of both the sensors is illustrated in the graphs below:

\section{Accuracy of Flex Sensor at different finger positions}
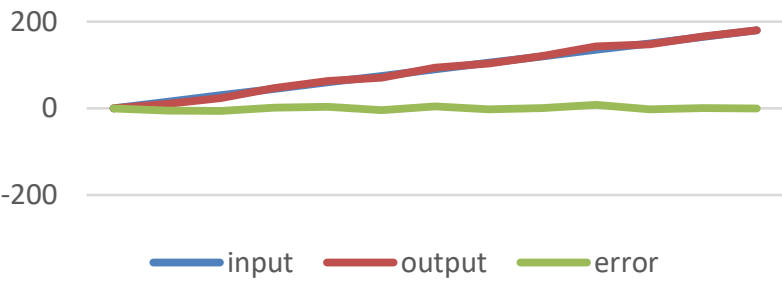

Fig. 6: (a) flex sensor accuracy analysis

\section{Accuracy of IMU over 50 Iterations}

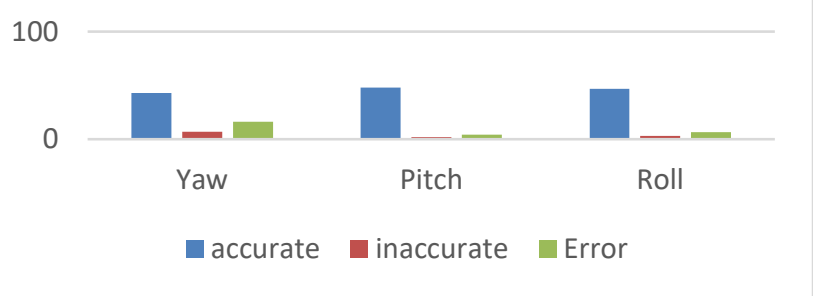

Fig. 6: (b) IMU accuracy analysis

\section{Conclusions}

The problem: (identification): Many wearable systems are being proposed by students in these emerging fields, but many of them fail to hit the market or the user domains, because of their size and price. It is easy enough to integrate the IMUs and other sensors available on the market together to form a wearable system, but the final product wouldn't be compact enough while the maker is restricted to semi-custom approach. Because of this the consumer is limited to choose either of the compactness and customizability while compromising the other.

The approach: (methodology): This problem is solved through fullcustom design of the components and circuit in a way that they could be easily embedded or printed on many wearable surfaces. Our project proposes a custom-made system with customizable networks where the user can keep, remove, use or detach components easily according to his comfort and specific requirement. Magnetic or metallic connectors make it easier and smoother, without the worry of connections getting damaged or worn out due to extensive usage and plug-ins and plug-outs. The proposed sensing system is essentially a kit of components which include multiple flex sensors to read finger gestures with Velcro straps, A wearable band with IMU to track arm and wrist motions, inbuilt wireless communication module, with additional GPIOs to further expand the applications (like EMG or EEG) and a portable li-ion battery pack, all packed into a flexible, wearable form factor. The actuator end that we plan to develop is theoretically a regular prosthetic arm using servos and lipo, but with extended capabilities of underwater propulsion and stereo-imaging units. This could be both wired or wireless depending upon the practical requirement.

Contribution to the community: Over the past few decades, Custom robotics and wearable electronics have gained an enormous popularity and attention, this has captured the interests of many hobbyists and enthusiasts and drawn them deeper into these fields, but the hardware resources are still quite an issue, though the open source microcontrollers and break-out boards are reliable these days, there is still a huge scope for further simplification and development in these fields.

Our project is a small contribution to the Oceanus community of developers, enthusiasts and hobbyists around the world which further increases the already extensively increasing scope of adopting 
machines, mechatronics and robotics, not only into industries but also into our individual lives to complete the regular tasks easier, or help accomplishing the tougher or sometimes super-human tasks in fields like disaster management and relief, which is one of our primary targets.

The robotic arms used by the government and NDRF for disaster management and relief these days are impractical in many practical situations because they lack gripping mechanisms and are also tough to control, through proper development of this project, we aim at fabricating an advanced arm, that perfectly mimics human hand gestures and movements, to simplify the control and extend the flexibility to higher degrees of motion. If not fabrication, even the design could serve as a step in the process of development of such advanced rescue equipment.

\section{Future Scope}

The results evidently prove the robust operation of the circuit, with the desired level of sensitivity and feasibility, thus could be developed further into a scaled down version, eliminating the unwanted and repeated components in the current circuit, using only core components like the IMU, AT Mega 328p, etc, instead of using the whole breakout boards that consume unwanted space.

This is an initial iteration for sensing flex, done with household materials, which contribute a certain level of non-linearity and noise. Since the iteration turned out to be successful, we now design the sensor with optimal performance abilities, along with a custom designed multi axis inertial measurement unit based on MEMS, as an extension, we also aim to develop the embedded single chip circuit for this sensing part, with all the flex sensors, IMU, processing unit, and wireless transceivers integrated onto a single, wearable chip, and further interface it with an actuating system, with servos, BLDCs and microcontrollers. Also, we desire to make the whole wearable system fully customizable with removable connections between the components, where the user is not limited to the original circuit itself, so that this system could be used as a stand - alone device that also supports few other necessary components in cases of emergencies or other such necessities. Besides, we could also make the system adaptable to various other sensor networks, and could extend its capabilities to work with electromyography, electroencephalography, and other futuristic under developing domains, to work even in situations where gestures couldn't be executed physically. In such conditions, the EMG or EEG signals could do the job, without the need of any actual physical movement or gesture.

\section{References}

[1] R. M. Edith and A. Bhargava Haripriya, "Gesture recognition using real time EMG," 2015 International Conference on Innovations in Information, Embedded and Communication Systems (ICIIECS), Coimbatore, 2015, pp. 1-3.

[2] C. Maj and A. Napieralski, "Mechanical simulation of 3-axis accelerometer using a single proof-mass," 2017 XIIIth International Conference on Perspective Technologies and Methods in MEMS Design (MEMSTECH), Lviv, 2017, pp. 47-50.

[3] H. Takahashi, S. Hirakawa, T. Takahata, K. Matsumoto and I. Shimoyama, "Quad-axial piezoresistive force sensor probe by four sensing elements with sidewall doping method," 2015 Transducers 2015 18th International Conference on Solid-State Sensors, Actuators and Microsystems (TRANSDUCERS), Anchorage, AK, 2015, pp. 1089-1092.

[4] F. Tounsi, E. Ayechi, B. Mezghani, M. Masmoudi, M. Kandpal and V. R. Rao, "Behavior analysis of a 3-axis detection push-pull piezoresistive MEMS accelerometer," 2015 IEEE 12th International Multi-Conference on Systems, Signals \& Devices (SSD15), Mahdia, 2015, pp. 1-6.
[5] A. Walther et al., "3-Axis gyroscope with $\mathrm{Si}$ nanogage piezoresistive detection," 2012 IEEE 25th International Conference on Micro Electro Mechanical Systems (MEMS), Paris, 2012, pp. 480483.doi: 10.1109/MEMSYS.2012.6170228.

[6] Dr. Seetaiah Kilaru, Hari Kishore K, Sravani T, Anvesh Chowdary L, Balaji T "Review and Analysis of Promising Technologies with Respect to fifth Generation Networks", 2014 First International Conference on Networks \& Soft Computing, ISSN:978-1-47993486-7/14,pp.270-273,August2014.

[7] Meka Bharadwaj, Hari Kishore "Enhanced Launch-Off-Capture Testing Using BIST Designs" Journal of Engineering and Applied Sciences, ISSN No: 1816-949X, Vol No.12, Issue No.3, page: 636643, April 2017.

[8] P Bala Gopal, K Hari Kishore, R.R Kalyan Venkatesh, P Harinath Mandalapu "An FPGA Implementation of On Chip UART Testing with BIST Techniques", International Journal of Applied Engineering Research, ISSN 0973-4562, Volume 10, Number 14 , pp. 34047-34051, August 2015

[9] A Murali, K Hari Kishore, D Venkat Reddy "Integrating FPGAs with Trigger Circuitry Core System Insertions for Observability in Debugging Process" Journal of Engineering and Applied Sciences, ISSN No: 1816-949X, Vol No.11, Issue No.12, page: 2643-2650, December 2016

[10] Mahesh Mudavath, K Hari Kishore, D Venkat Reddy "Design of CMOS RF Front-End of Low Noise Amplifier for LTE System Applications Integrating FPGAs" Asian Journal of Information Technology, ISSN No: 1682-3915, Vol No.15, Issue No.20, page: 4040-4047, December 2016.

[11] N Bala Dastagiri, Kakarla Hari Kishore "Reduction of Kickback Noise in Latched Comparators for Cardiac IMDs" Indian Journal of Science and Technology, ISSN No: 0974-6846, Vol No.9, Issue No.43, Page: 1-6, November 2016

[12] S Nazeer Hussain, K Hari Kishore "Computational Optimization of Placement and Routing using Genetic Algorithm" Indian Journal of Science and Technology, ISSN No: 0974-6846, Vol No.9, Issue No.47, page: 1-4, December 2016.

[13] Meka Bharadwaj, Hari Kishore "Enhanced Launch-Off-Capture Testing Using BIST Designs" Journal of Engineering and Applied Sciences, ISSN No: 1816-949X, Vol No.12, Issue No.3, page: 636643, April 2017.

[14] N Bala Dastagiri,, K Hari Kishore "Analysis of Low Power Low Kickback Noise in Dynamic Comparators in Pacemakers" Indian Journal of Science and Technology, ISSN No: 0974-6846, Vol No.9, Issue No.44, page: 1-4, November 2016.

[15] S.V.Manikanthan and T.Padmapriya "Recent Trends In $\mathrm{M} 2 \mathrm{~m}$ Communications In 4g Networks And Evolution Towards 5g", International Journal of Pure and Applied Mathematics, ISSN NO:1314-3395, Vol-115, Issue -8, Sep 2017.

[16] T. Padmapriya and V.Saminadan, "Improving Performance of Downlink LTE-Advanced Networks Using Advanced Networks Using Advanced feedback Mechanisms and SINR Model", International Conference on Emerging Technology (ICET), vol.7, no.1, pp: 93, March 2014.

[17] Meka Bharadwaj, Hari Kishore "Enhanced Launch-Off-Capture Testing Using BIST Designs" Journal of Engineering and Applied Sciences, ISSN No: 1816-949X, Vol No.12, Issue No.3, page: 636643, April 2017. 